Gynecol Obstet Invest 1995;39:281-282

\title{
Author Index Vol. 39, 1995
}

Aberg, H. 195 Achiron,R. 151 Aitken, M.A. 73 Aloysio, D. de 115 Altieri,P. 115 Anyaegbunam, A.M. 165 Ariely,S. 143 Aso,T. 190 Ausch,Ch. 11 Axelsson, O. 120 Axemo, P. 103 Azim, F.A. 97

Barad,D.H. 43 Barbieri, R.L. 47,266 Bauer, K. 11 Ben-Ami, M. 50 Ben-Ezra, J. 277

Bergström,S. 103,178,252 Bhuiyan, A.B. 97 Biagini,G. 239 Bilgin,T. 184 Bique,C. 252 Bjøro, K. 8 Blann,E. 28 Bledsoe,L.B. 79 Blombäck,M. 234 Bracero, L.A. 221 Brandenburg, H. 147 Bremme, K. 234 Brennecke, S.P. 73 Bruijn, H.W.A. de 147 Bruschi, F. 63 Bugalho,A. 252 Bukovsky,I. 143,217 Buhner, J.N. 155 Byström,B. 195

Carretti,N. 83 Caruana, P. 213 Castaldini, C. 239 Cengiz,C. 184 Cester,N. 239 Chalubinski, K. 24 Check, J.H. 257 Ciavattini, A. 130 Cohen, B.L. 43 Crosignani, P.G. 63 Cunningham, D.S. 54,79

Daenens, P. 110 Darj,E. 120 Demir,U. 184

De Nictolis, M. 130 Direm,B. 229 Divers, M.J. 155 Dogan, M. 60 Dowell,R.T. 247

Drijkoningen, M.P.G. 34 Dweck,H.S. 221

Ekici,E. 60 El Maradny, E. 1,97 Eppel,W. 210 Eremita,G.A. 83 Eyali,V. 50

Fabris,N. 130 Feltmate,C. 47 Folgosa, E. 178 Forsberg, A.L. 247 Frable,WJ. 277 Frank, A. 210 Freed, K.A. 73 Frigo,P. 210 Fujino,Y. 205 Fujisawa, K. 274 Fung,H.Y.M. 262 Furuhashi,N. 88 Gandy,P. 257 Gao,X. 47 Garzetti, G.G. 130 Gebre-Medhin, M. 103 Geppert, M. 136 Geslevich,Y. 50 Gholbzouri, K. 274 Ginsburg, E.S. 47,266 Gleason,R.E. 47 Glover, D.D. 67 Gökmen,O. 60,229 Golan, A. 217 Golaszewski, T. 210 Goldman, B. 151 Goteri,G. 130 Gregoriou, O. 226 Grella,P. 83 Grin,W. 65 Gruber,W. 24,210 Grünberger, W. 65 Hadas,E. 217 Halim,A. 1,97 Halperin,R. 143,217 Hasegawa, N. 125 Hata,K. 70 Hata,T. 70,169 Haugen, G. 8 Hayashi, M. 141 He, S. 234 Herman, A. 143,217 Hiroshi,K. 97 Huber,K. 11 Huber,M. 65

Ide,P.S. 34,110 Ishihara,H. 39 Ishikawa, H. 125 Itamochi, H. 39 Itoi,H. 15 Janse,H.C. 147

Kaleli,B. 60 Kallner,A. 234 Kanamori, T. 141 Kanayama, N. 1,97 Karayalçin, R. 60 Katz,Z. 271 Kawai, M. 125 Kay,S. 277 Khatun,S. 97 Kieikowski, A. 93 Kigawa, J. 39 Kikkawa,F. 125 Kimura, H. 88 Kitao,M. 70, 169, 199 Klein, M.J. 11 Konidaris, S. 226 Kornstein, M.J. 277 Kristiansson, P. 195 Kubota,T. 190 Kujansuu, E. 186 Kuraya,K. 160 Langer, O. 19 Larsen, B. 67 Lauer,C. 257 Lauweryns, J.M. 34 Levy,R. 271 Libombo, A. 178 Lilford,RJ. 155 Liljestrand, J. 103 Lipitz, S. 151 Los,FJ. 147 Lucarini, G. 239 Lurie, S. 271 Machungo, F. 252 Maehara, K. 1,97 Malek,A. 28 Manabe,A. 70, 169 Mancuso, S. 213 Marana,R. 213 Maruyama, H. 160 Mashiach, S. 151 Matilsky, M. 50 Matsuda,Y. 160 Matsumura, M. 141 Matsushita, K. 141 Mattison, D.R. 28 Maymon, R. 143 Mazzanti, L. 239 Menso, S. 130 Meschia, M. 63 Mikhail, M.S. 165 Miller, D. 155 Minagawa, Y. 39 Minakami, H. 15 Miyakawa, I. 274 Mizuno, K. 125 Moroz,Ch. 11 Muzii, L. 213 Muzzioli,M. 130 Nagae,H. 88 Nagami, M. 39 Nilsson, G. 120 Nilsson,S. 120 Nonogaki,T. 141 
Oga,M. 274 Ogita, S. 205 Okada,M. 199 Olechowski, M. 65 Opsahl,M.S. 79 Ozaki,T. 199 Özcan, T. 60,229

Palan,P.R. 43 Paltrinieri, F. 115 Papadias, C. 226 Parazzini, F. 63 Paternoster, D. 83

Peeters, R. 110 Pizzichini, M. 83 Ploeckinger, B. 24 Poppe,W.AJ. 34,110 Pretolani,G. 115

Pugnaloni, A. 239 Pyrgiotis, E. 226

Reale,M.R. 221 Redlich,K. 11 Reubinoff, B.E. 19 Rice,G.E. 73 Risberg,B. 120 Rogers, M.S. 262 Romanini, C. 239 Romeo, A. 115 Romney, S.L. 43 Ron-El, R. 143,217 Rosen, A.C. 11 Rosen, H.R. 11

Sahin,N. 229 Sakata,Y. 15 Salvolini,E. 239 Samueloff, A. 19 Sato, I. 15 Scarpelli, S. 165

Schneider, D. 217 Schoultz, B. von 195 Senöz,S. 229 Shah,D.T. 67 Shalev,E. 50 Shea,B.F. 47

Shimoyama, I. 1 Shintaku,Y. 141 Smyczek-Gargya, B. 136 Staffolani, R. 239 Stastny,J.F. 277

Stewart, J.A. 277 Stray-Pedersen, S. 8 Sumimoto, K. 1

Taguchi,M. 190 Takahashi,K. 199 Takai,N. 274 Takebayashi, T. 205

Tamada, T. 15 Tamakoshi, K. 125 Tanasijevic, M.J. 266 Tarquini, P. 257 Tauchi,K. 141

Te,M.N. 221 Terakawa, N. 39 Terao,T. 1,97 Tichenor, J.R. 79 Tietz,C. 239 Tinkanen, H. 186

Tomoda,Y. 125 Turan,C. 60

Uchida,A. 199 Ulm,M.R. 24,210 Uludag,H. 60 Umesaki,N. 205 Uszyíski, M. 93 Uszyíski,W. 93

Van Assche, F.A. 34, 110 Vrij,R.W.de 147

Walsh, B.W. 47 Watanabe, T. 15 Weinraub,Z. 143 Weinstein,D. 19 Weissman, A. 151 Wierrani, F. 65 Winkelman, J.W. 266 Wong,F.W.S. 262 Wong,Y.L. 262 Wreje,U. 195 Wybenga,D.R. 266

Yajima,A. 88 Yamasaki,H. 199 Yoshida,M. 141 Yoshimatsu, J. 274 Yura,Y. 141

Zeballos, G.A. 221 Zourlas, P.A. 226

282

Author Index 